\title{
Massive Hemoptysis Due to the Rupture of Thoracic Aortic Aneurysm Caused by Leukemic Cell Infiltration in a Patient With Chronic Myelomonocytic Leukemia
}

\author{
Hiroaki Tanaka $^{\mathrm{a}, \mathrm{e}}$, Mizuki Yano ${ }^{\mathrm{b}}$, Chihiro Kuwabara ${ }^{\mathrm{a}}$, Ayaka Kume ${ }^{\mathrm{a}}$, Yuri Tamura ${ }^{\mathrm{a}}$, \\ Miki Murakamic, Ryo Shimizu ${ }^{\mathrm{a}}$, Haruhisa Saito ${ }^{\mathrm{d}}$, Yoshio Suzukic
}

\begin{abstract}
Hemoptysis is occasionally experienced in patients with hematological malignancies who have respiratory tract infection and severe thrombocytopenia. Thrombocytopenia due to hematological disease is one cause of hemoptysis. Chronic myelomonocytic leukemia (CMML) is a clonal hematopoietic malignancy characterized by both a myeloproliferative neoplasm and a myelodysplastic syndrome. This malignancy often infiltrates various extramedullary organs and has a poor prognosis. An 84-year-old Japanese man with CMML was suffered from hemoptysis and dyspnea. When he arrived at the emergency room, hemoptysis stopped. His white blood cell count was 866 $\times 10^{9} / \mathrm{L}$ with $3.5 \%$ blast cells and $36.5 \%$ monocytes; hemoglobin was $6.7 \mathrm{~g} / \mathrm{dL}$; platelets count was $19 \times 10^{9} / \mathrm{L}$; and C-reactive protein was $16.23 \mathrm{mg} / \mathrm{dL}$. Chest X-ray examination revealed an invasion shadow near the mediastinum in the left upper lung field. Chest computed tomography revealed a tumorous lesion in the left upper lobe, which had progressed to the mediastinum and formed an infiltration shadow around it. He was administered the antibiotics and the hemostatic agents under hospitalization. He also received blood transfusion for anemia and thrombocytopenia. Rapid improvement in oxygenation was observed along with a rapid decrease in blood levels in the sputum. On the eighth days of hospitalization, however, the patient newly developed massive hemoptysis and died. Autopsy revealed rupture of a thoracic pseudoaneurysm due to infiltration of leukemia cells in the tunica media and lung. Clinicians should consider thoracic aortic aneurysms as a possible cause of hemoptysis even in cases with small hemoptysis. It should be noted that in CMML patients, direct infiltration of leukemia cells in the vascular wall can cause aneurysm formation.
\end{abstract}

Keywords: Hemoptysis; Chronic myelomonocytic leukemia; Tho-

Manuscript submitted December 8, 2018, accepted December 21, 2018

aDepartment of Hematology, Asahi General Hospital, Chiba, Japan bepartment of Internal Medicine, Asahi General Hospital, Chiba, Japan 'Department of Clinical Pathology, Asahi General Hospital, Chiba, Japan dDepartment of Respiratory Medicine, Asahi General Hospital, Chiba, Japan ${ }^{e}$ Corresponding Author: Hiroaki Tanaka, Department of Hematology, Asahi General Hospital, I-1326, Asahi-city, Chiba 289-2511, Japan.

Email: htanaka@hospital.asahi.chiba.jp

doi: https://doi.org/10.14740/jocmr3712 racic aortic aneurysm; Rupture of aneurysm

\section{Introduction}

Hemoptysis is occasionally experienced in patients with hematological malignancies who have respiratory tract infection and severe thrombocytopenia. Thrombocytopenia due to hematological disease is one cause of hemoptysis [1].

Chronic myelomonocytic leukemia (CMML) is a clonal hematopoietic malignancy characterized by both a myeloproliferative neoplasm and a myelodysplastic syndrome; this malignancy often infiltrates various extramedullary organs and has a poor prognosis [2]. Although allogeneic stem cell transplantation is the only treatment that can cure CMML, azacitidine therapy can extend the survival period of these patients [3].

Here we describe a case with CMML, who developed massive hemoptysis due to the rupture of a thoracic aortic aneurysm caused by leukemic cell infiltration.

\section{Case Report}

In April 2017, an 84-year-old Japanese man suffering from dyspnea and appetite loss was referred to our hospital. He had undergone appendectomy at 34 years of age and used to smoke 10 cigarettes a day until before being referred to our hospital. Laboratory findings at the referred to our hospital were as follows: white blood cells, $42.8 \times 10^{9} / \mathrm{L}$ with $16 \%$ blast cells and $38 \%$ monocytes; hemoglobin, $6.2 \mathrm{~g} / \mathrm{dL}$; platelets, $35 \times 10^{9} / \mathrm{L}$; lactate dehydrogenase, $1,541 \mathrm{U} / \mathrm{L}$; and C-reactive protein, 1.70 $\mathrm{mg} / \mathrm{dL}$. Bone marrow examination revealed hypercellularity with decreased megakaryocytes and increased monocytes (Table 1). Forty percent of megakaryocytes had multiple, widelyseparated nuclei and $10 \%$ of erythrocytes had megaloblastoid change (Fig. 1). Chromosomal analysis of bone marrow specimens using G-band staining was normal. The patient was diagnosed with CMML-2 and treated by $75-\mathrm{mg} / \mathrm{m}^{2}$ azacitidine for 7 days every 4 weeks. At the same time, the patient was diagnosed with chronic obstructive lung disease by spirograph and chest computed tomography (CT) and treated with tiotro- 
Table 1. Bone Marrow Examination at the Referred to Our Hospital

\begin{tabular}{ll}
\hline Bone marrow examination & \\
NCC & $2,013 \times 10^{9} / \mathrm{L}$ \\
Mgk & $0.06 \times 10^{9} / \mathrm{L}$ \\
Basophilic erythroblast & $2.0 \%$ \\
Polychromatic erythroblast & $7.2 \%$ \\
Orthochromatic erythroblast & $1.4 \%$ \\
Myeloblast & $9.0 \%$ \\
Promyelocytes & $1.2 \%$ \\
Myelocytes & $25.6 \%$ \\
Metamyelocytes & $2.8 \%$ \\
Stab cells & $3.8 \%$ \\
Segmented cells & $0.2 \%$ \\
Eosinophil & $0.2 \%$ \\
Promonocytes & $3.4 \%$ \\
Monocytes & $27.2 \%$ \\
Lymphocytes & $2.6 \%$ \\
\hline
\end{tabular}

pium/olodaterol. Soon after undergoing azacitidine therapy, blast cells in the peripheral blood decreased to $1-2 \%$. However, the therapy was discontinued after two courses on the patient's request.
In August 2017, the patient had bled from the oral cavity and the nose. In the morning 2 days after that, he suffered from hemoptysis and dyspnea and was thus transported to our hospital via an ambulance. When he arrived at the emergency

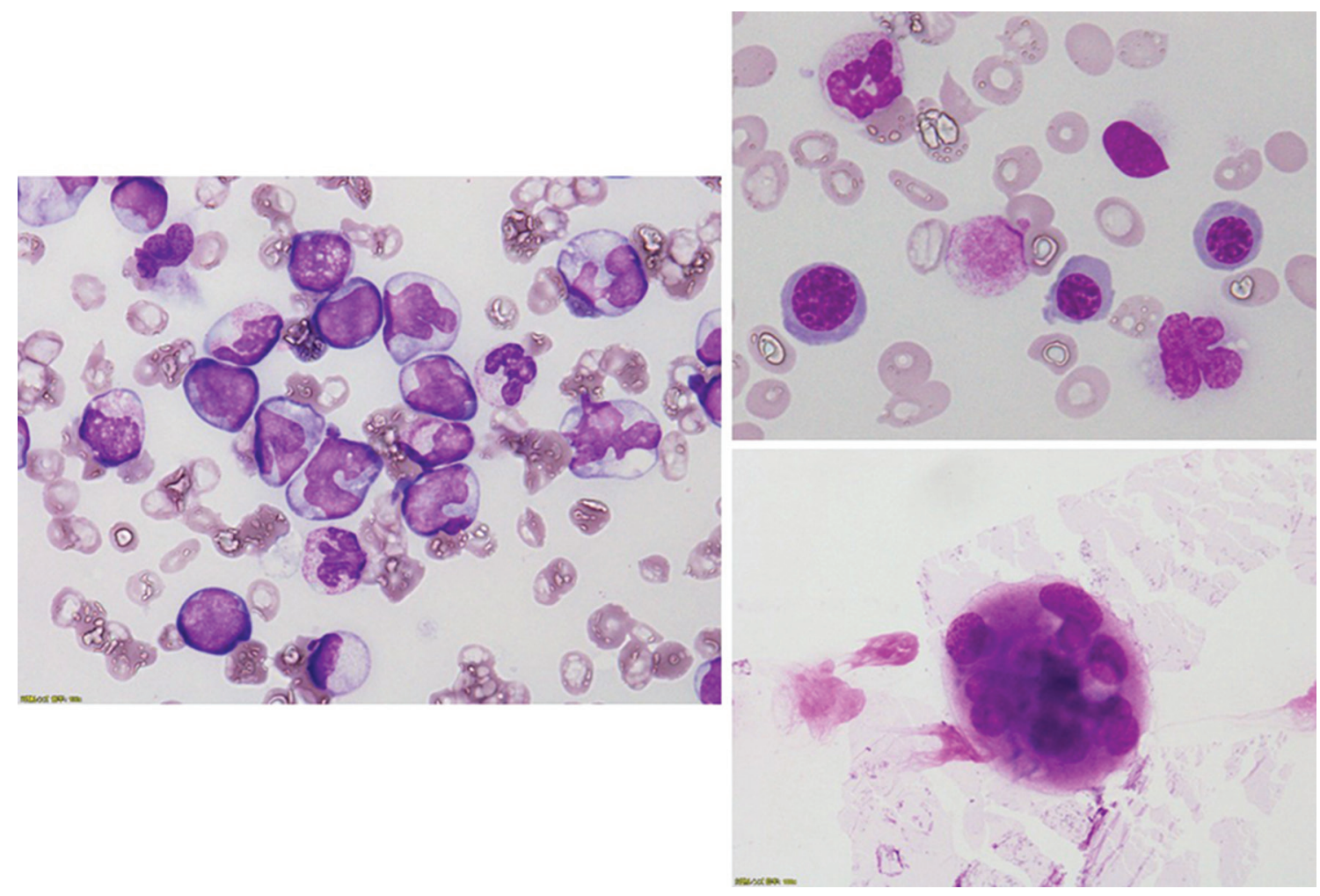

Figure 1. Bone marrow examination revealed a hypercellular bone marrow with decreased megakaryocytes and increased monocytes. Forty percent of megakaryocytes had multiple, widely-separated nuclei and $10 \%$ of erythrocytes had megaloblastoid change. 
Table 2. Laboratory Data on Admission

\begin{tabular}{|c|c|}
\hline \multicolumn{2}{|l|}{ Complete blood cell count } \\
\hline White blood cell & $866 \times 10^{9} / \mathrm{L}$ \\
\hline Blast & $3.5 \%$ \\
\hline Myelocyte & $18.5 \%$ \\
\hline Metamyelocytes & $7.5 \%$ \\
\hline Stab cells & $1.5 \%$ \\
\hline Segmented cells & $25.0 \%$ \\
\hline Lymphocyte & $7.5 \%$ \\
\hline Monocyte & $36.5 \%$ \\
\hline Hemoglobin & $6.7 \mathrm{~g} / \mathrm{dL}$ \\
\hline Platelet count & $19 \times 10^{9} / \mathrm{L}$ \\
\hline \multicolumn{2}{|l|}{ Coagulation test } \\
\hline PT & $17.0 \mathrm{~s}$ \\
\hline PT-INR & 1.38 \\
\hline APTT & $44.8 \mathrm{~s}$ \\
\hline Fibrinogen & $533 \mathrm{mg} / \mathrm{dL}$ \\
\hline FDP & $10.8 \mu \mathrm{g} / \mathrm{mL}$ \\
\hline \multicolumn{2}{|l|}{ Blood chemistry/serological test } \\
\hline Total protein & $7.1 \mathrm{~g} / \mathrm{dL}$ \\
\hline Albumin & $2.6 \mathrm{~g} / \mathrm{dL}$ \\
\hline Aspartate transaminase & $67 \mathrm{U} / \mathrm{L}$ \\
\hline Alanine aminotransferase & $39 \mathrm{U} / \mathrm{L}$ \\
\hline Lactate dehydrogenase & $929 \mathrm{U} / \mathrm{L}$ \\
\hline Alkaline phosphatase & $737 \mathrm{U} / \mathrm{L}$ \\
\hline Gamma-glutamyl transpeptidase & $114 \mathrm{U} / \mathrm{L}$ \\
\hline Total bilirubin & $0.7 \mathrm{mg} / \mathrm{dL}$ \\
\hline Uric acid & $4.6 \mathrm{mg} / \mathrm{dL}$ \\
\hline Urea nitrogen & $60 \mathrm{mg} / \mathrm{dL}$ \\
\hline Creatinine & $3.00 \mathrm{mg} / \mathrm{mL}$ \\
\hline Creatine phosphokinase & $35 \mathrm{U} / \mathrm{L}$ \\
\hline C-reactive protein & $16.23 \mathrm{mg} / \mathrm{dL}$ \\
\hline \multicolumn{2}{|l|}{ Infection-associated test } \\
\hline Blood culture (2 set) & Negative \\
\hline Sputum culture & Resident bacteria of the oral cavity \\
\hline Gaffky scale (3 set) & 0 \\
\hline
\end{tabular}

APTT: activated partial thromboplastin; PT: prothrombin time; INR: international normalized ratio; FDP: fibrinogen degradation product; F: coagulation factor.

room, hemoptysis stopped. His vital signs were as follows: temperature, $38.1^{\circ} \mathrm{C}$; heart rate, 146 beats $/ \mathrm{min}$; blood pressure, 119/64 mm Hg; and oxygen saturation, $88 \%$ under 10 L oxygen administered through an oxygen mask with a reservoir bag. Physical examination revealed decreased respiratory sounds in his left upper lung but no rale or abnormal heart sounds. The liver, spleen and superficial lymph nodes were unpalpable. Petechial bleeding was observed in the chest, abdo- men and limbs, but no oral bleeding was observed. Laboratory findings at the emergency room were showed in Table 2. Chest $\mathrm{X}$-ray examination revealed an invasion shadow near the mediastinum in the left upper lung field (Fig. 2a). Chest plain CT revealed a tumorous lesion in the left upper lobe. This mass had progressed to the mediastinum and formed an infiltration shadow around it (Fig. 2b). Due to the patient's renal disorder, enhanced CT could not be performed. 

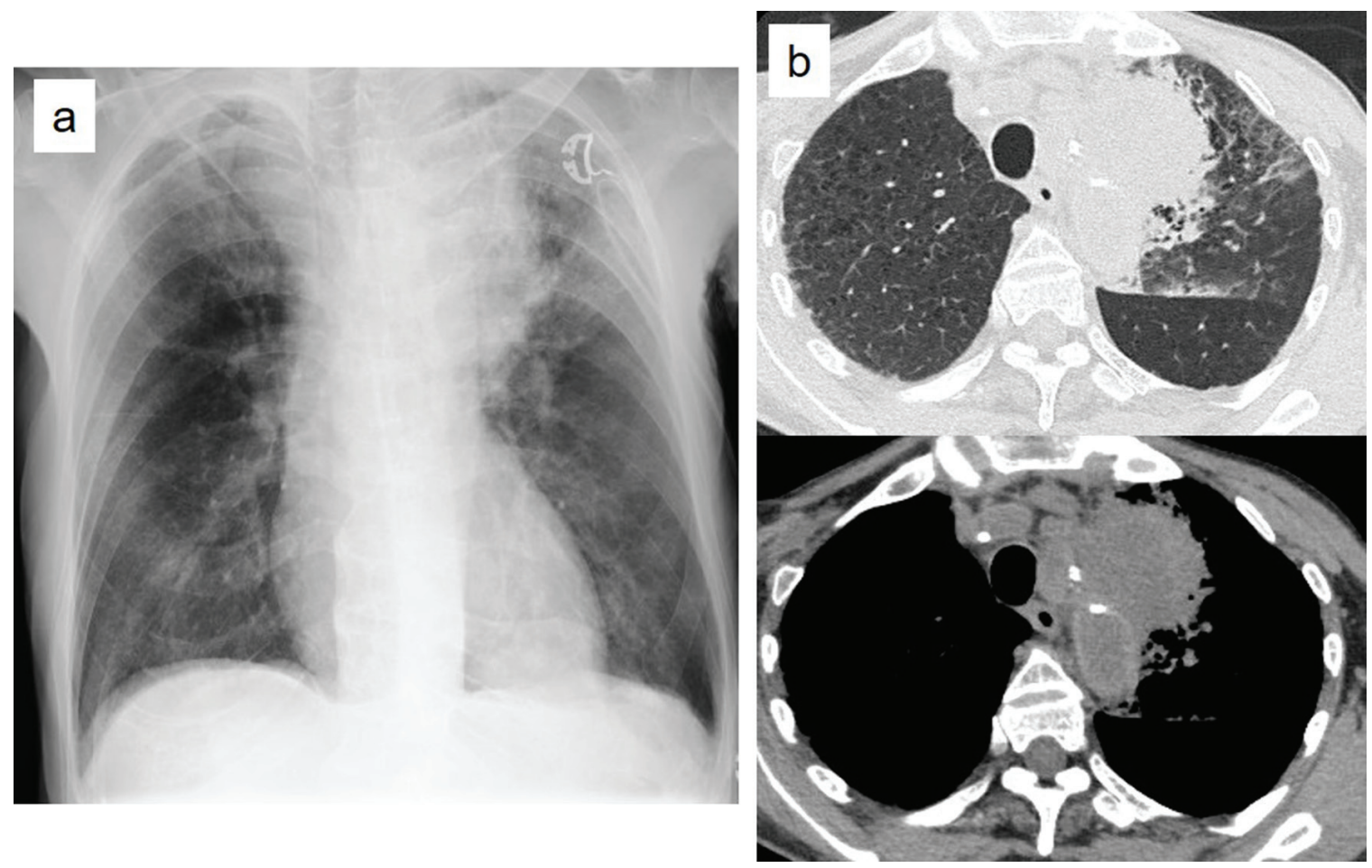

Figure 2. Chest X-ray examination revealed an invasion shadow near the mediastinum of the left upper lung field (a). Chest plain computed tomography revealed a tumorous lesion in left upper lobe, which progressed to the mediastinum and formed an infiltration shadow around it (b).

Patient's oxygen administration was continued, and he was administered the antibiotics ampicillin/sulbactam and the hemostatic agents carbazochrome sodium sulfonate hydrate and tranexamic acid. He also received blood transfusion foranemia and thrombocytopenia. Improvement in oxygenation was observed in the same afternoon, along with a rapid decrease in blood levels in the sputum. On the seventh day of hospitalization, oxygen administration was discontinued. However, in the early morning of the eighth day of hospitalization, the patient newly developed massive hemoptysis, went into cardiopulmonary arrest and died.

Autopsy was performed after obtaining the consent of his family. It revealed that the cellular bone marrow was filled with homogeneous leukemia cells in the form of acute myeloid leukemia (Fig. 3a, b). Infiltration of leukemia cells in the lung, spleen, liver, kidney and lymph nodes was also noted. A saccular aneurysm of $2.5 \mathrm{~cm}$ diameter was identified in the descending aortic arch. It was adhered to the left upper lobe and collapsed, forming a pseudoaneurysm on the lung side (Fig. $3 \mathrm{c}, \mathrm{d})$. This pseudoaneurysm had bled and coagulated. In the aorta, infiltration of leukemia cells in the tunica media and atherosclerotic changes were observed (Fig. 3e-g). Infiltration of leukemia cells was also observed in the lungs near the ruptured area (Fig. 3h, i).

\section{Discussion}

Major causes of hemoptysis are chronic inflammatory lung dis- eases and bronchogenic carcinoma [1, 4]. Massive hemoptysis accounts for only $1.5 \%$ of all hemoptysis cases and predominantly occurs in cases of tuberculosis, carcinoma, fungal ball and bronchial communication with a leaky aortic aneurysm [4]. The mortality rate of patients with massive hemoptysis is $38-50 \%[1,5]$. In our case, hemoptysis at the time of hospitalization was small. Therefore, we considered it to have been due to a bleeding tendency caused by severe thrombocytopenia and pneumonia. However, if the chest plain CT findings could be correctly interpreted considering thoracic aneurysm to be a cause of the hemoptysis, our patient would have been saved by prompt treatment. Clinicians should consider thoracic aortic aneurysms as a possible cause of hemoptysis even in cases of small hemoptysis.

The most notable point in our case is that the formation and rupture of the thoracic aortic aneurysm was caused by the infiltration of leukemia cells into the tunica media and lung.

In patients with CMML, systemic autoimmune and/or inflammatory diseases are frequently observed, the most common of which is systemic vasculitis [6-10]. There were some reports describing aneurysm rupture following vasculitis in case with CMML $[11,12]$. In our case, however, systemic vasculitis was not considered as a possible cause because of the absence of abnormal findings in the remaining vasculature. To the best of our knowledge, case reports describing the rupture of an aneurysm due to direct infiltration of leukemia cells in the vascular wall are extremely rare [13-15]. To date, only one case report of a CMML patient has been published, describing a massive hemoptysis following a pulmonary artery aneurysm 

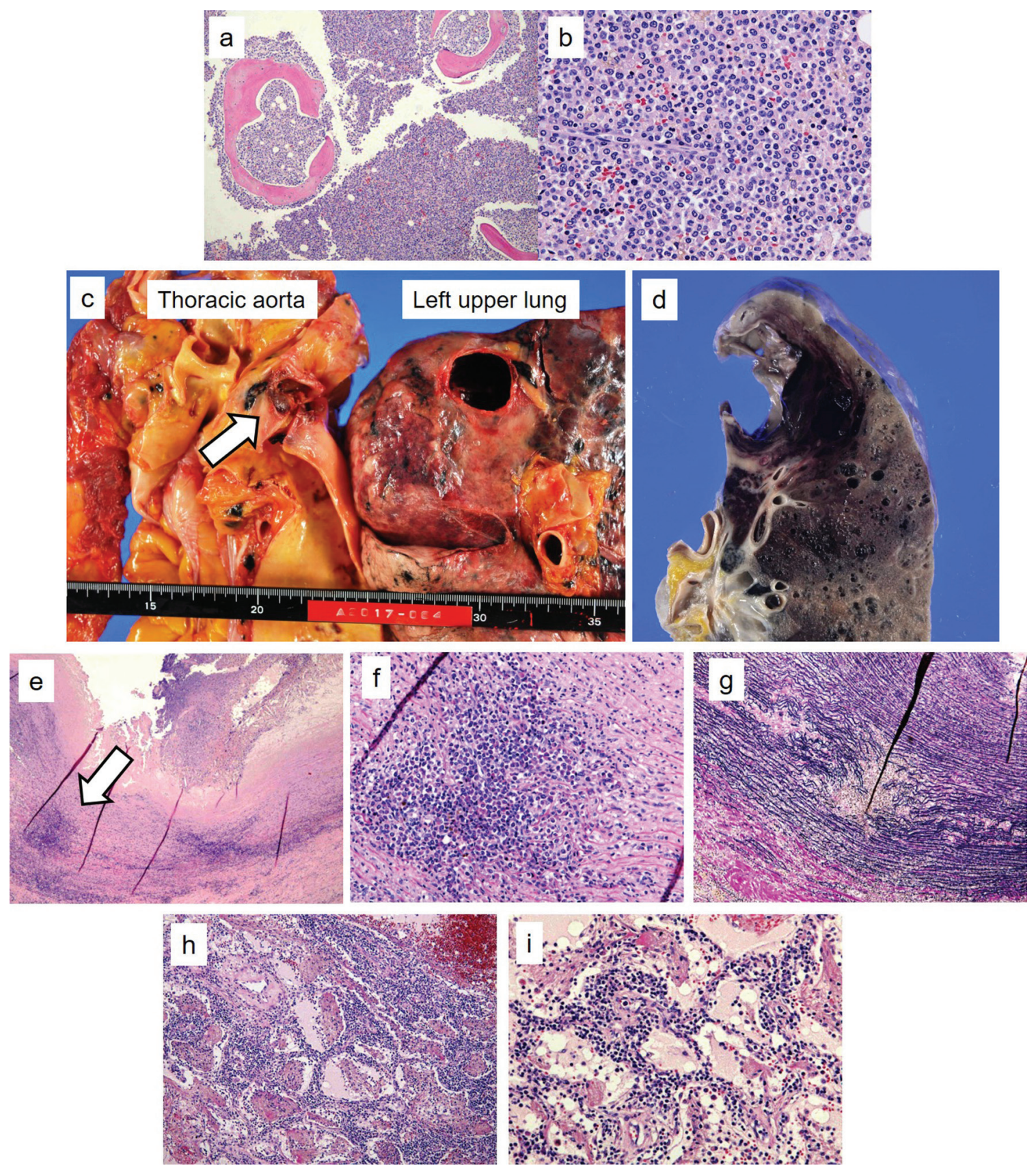

Figure 3. Autopsy revealed that the cellular bone marrow was filled with homogeneous leukemia cells in the form of acute myeloid leukemia ((a) hematoxylin and eosin staining, $\times 100$; (b) hematoxylin and eosin staining, $\times 400)$. A saccular aneurysm of 2.5 $\mathrm{cm}$ in diameter was found in the descending aortic arch. It was adhered to the left upper lobe and collapsed (arrow, c), forming a pseudoaneurysm on the lung side (d). In the aorta, infiltration of leukemia cells in the tunica media was observed, along with atherosclerotic changes ((e) arrow, hematoxylin and eosin staining, $\times 100$; (f) hematoxylin and eosin staining, $\times 400$; (g) Elastica van Gieson staining $\times 400)$. Infiltration of leukemia cells was also observed in the lungs near the ruptured area ((h) hematoxylin and eosin staining, $\times 100$; (i) hematoxylin and eosin staining, $\times 400$ ).

caused by the infiltration of leukemia cells in the pulmonary tissue surrounding the aneurysm [15]. However, there may be other CMML patients who have died of rupture of aneurysm due to the direct infiltration of leukemia cells without correct diagnosis because rupture of an aneurysm is mostly fatal. Furthermore, among these patients, some may have been in- correctly diagnosed with systemic vasculitis because in most patients with vasculitis-associated CMML, tests for antineutrophil cytoplasmic antibodies are negative [7].

In conclusion, here we reported the case of a CMML patient who developed massive hemoptysis due to thoracic aortic aneurysm rupture caused by leukemic cell infiltration. Clini- 
cians should consider thoracic aortic aneurysms as a possible cause of hemoptysis even in cases with small hemoptysis. It should be noted that in CMML patients, in addition to systemic vasculitis, direct infiltration of leukemia cells in the vascular wall can also cause aneurysm formation.

\section{Conflict of Interest}

All the authors declare no conflict of interest.

\section{References}

1. Hirshberg B, Biran I, Glazer M, Kramer MR. Hemoptysis: etiology, evaluation, and outcome in a tertiary referral hospital. Chest. 1997;112(2):440-444.

2. Orazi A BJ, Germing U, Brunning RD, et al. Chronic myelomonocytic leukaemia. In: Swerdlow SH, Campo E, Harris NL, et al. editors. WHO classification of tumours of haematopoietic and lymphoid tissues. Revised 4th ed. Lyon: IARC; 2017. p. 82-86.

3. Patnaik MM, Tefferi A. Chronic myelomonocytic leukemia: 2018 update on diagnosis, risk stratification and management. Am J Hematol. 2018;93(6):824-840.

4. Jean-Baptiste E. Clinical assessment and management of massive hemoptysis. Crit Care Med. 2000;28(5):16421647.

5. Khalil A, Fedida B, Parrot A, Haddad S, Fartoukh M, Carette MF. Severe hemoptysis: From diagnosis to embolization. Diagn Interv Imaging. 2015;96(7-8):775788 .

6. Grignano E, Mekinian A, Braun T, Liozon E, Hamidou M, Decaux O, Puechal X, et al. Autoimmune and inflammatory diseases associated with chronic myelomonocytic leukemia: A series of 26 cases and literature review. Leuk Res. 2016;47:136-141.
7. Hamidou MA, Boumalassa A, Larroche C, El Kouri D, Bletry O, Grolleau JY. Systemic medium-sized vessel vasculitis associated with chronic myelomonocytic leukemia. Semin Arthritis Rheum. 2001;31(2):119-126.

8. Park JK, Gelber AC, Zheng G, McDevitt MA, Gocke CD, Baer AN. Large-vessel vasculitis as an early manifestation of chronic myelomonocytic leukemia. J Clin Oncol. 2011;29(20):e601-603.

9. Mori M, Togami K, Fujita H, Inoue D, Kimura T, Shimoji S, Nagai Y, et al. Successful allogeneic bone marrow transplantation for chronic myelomonocytic leukemia complicated by refractory aortitis. Bone Marrow Transplant. 2010;45(4):796-797.

10. Sasinowska S, Traisak P, McCormack M, Eid H. A rare case of paraneoplastic aortitis associated with chronic myelomonocytic leukemia. Case Rep Hematol. 2017;2017:3091973.

11. Jeanmonod R, Lewis C. Bilateral renal artery aneurysm rupture in a man with leukemia: report of a case. J Vasc Surg. 2008;47(4):871-873.

12. Georgiou C, Krokidis M, Elworthy N, Dimopoulos S. Spontaneous bilateral renal aneurysm rupture secondary to Polyarteritis Nodosa in a patient with chronic myelomonocytic leukaemia: A case report study. Int J Surg Case Rep. 2016;26:61-64.

13. Shifrin EG, Drenger B, Matzner Y, Kaplan L. Ruptured inflammatory abdominal aortic aneurysm due to acute myelomonoblastic leukemia. J Cardiovasc Surg (Torino). 1987;28(1):32-34.

14. Pontailler M, Fabre D, Hocquemiller-Khalife T, Fadel E. Thoraco-abdominal aortic aneurysm rupture secondary to lymphocytic lymphoma. Interact Cardiovasc Thorac Surg. 2017;24(1):156-157.

15. Hamamoto F, Takahashi T, Hayashi T, Somekawa T, Hosokawa A, Ito Y, Adachi M, et al. [Localized pulmonary infiltration in chronic myelomonocytic leukemia]. Rinsho Ketsueki. 1998;39(3):205-209. 